\title{
CLINICAL NOTES \\ Management of end-stage kidney failure in patients with adult polycystic kidney disease
}

\author{
Tunde Campbell \\ Consultant Transplant Surgeon, Kidney \& Pancreas Transplant Centre, Manchester University Hospitals NHS \\ Foundation Trust, Manchester Royal Infirmary, Oxford Road, Manchester, United Kingdom
}

Keywords: Kidney, Polycystic; Genetic; Autosomal; Nephrectomy; Transplant

\section{Introduction}

1 dult polycystic kidney disease (APKD) is a genetic inherited disorder characterised by enlarging cysts in the kidney which compress and damage it, resulting over time to kidney enlargement, dysfunction, urological complications and eventual failure. Most patients may have several cysts in the liver, pancreas and spleen, which rarely damage their function. The commoner type of APKD is the autosomal dominant variant PKD1 (ADPKD), which affects $90 \%$ of patients. The autosomal recessive variant affects $10 \%$ of patients. The child who has a parent with ADPKD has a 50\% chance of inheriting the disease, and $100 \%$ if both parents have ADPKD. In sporadic genetic mutations, neither parent has the disease, and this occurs in about $10 \%$ of patients. About $70 \%$ of individuals will develop renal failure at the age of 65 years, and dialysis will be required in $50 \%$ of individuals at the age of 60 years. Affected individuals do not often experience symptoms until they are 30-50 years old and, in a few, even after the age of 70-80 years.

APKD is a worldwide problem affecting all races. It is the fourth leading cause of end-stage kidney disease in adults. In the UK, it affects 1 in 800 people in the population. In the USA, ADPKD is the cause of end-stage renal failure (ESRF) requiring dialysis in $1.4 \%$ of Black people and $6.8 \%$ of Caucasian people. In Europe, it is the cause of ESRF in 6-10\% of all dialysis patients. Patients with ADPKD tend to experience better survival on dialysis compared to patients with other causes of ESRF. The disease has not been known to recur in the transplant kidney unlike other kidney diseases such as focal segmental glomerulosclerosis (FSGS), membranoproliferative glomerulonephritis (MPGN), immunoglobulin A (IgA) nephropathy (Berger's disease) and membranous nephropathy (MN). Extra renal associated conditions include a higher risk of intra-cranial berry aneurysms $(8 \%)$, hepatomegaly, thoracic aortic aneurysm and heart valve problems, while $80 \%$ of patients may experience diverticulosis while on dialysis.

Early symptoms include back and loin ache, haematuria (blood in the urine), headache, swelling of the abdomen and high blood pressure. The clinical presentation, onset of and severity of symptoms vary. A proportion of patients with ADPKD will present with a subarachnoid haemorrhage (SAH) before renal abnormalities are obvious. Patients with ADPKD tend to start dialysis earlier, median age 55 years, than patients whose kidneys failed from other causes (median age 66 years). Other presentations may include urological complications such as urinary tract infection, kidney stones (rare), bleeding into the cysts, recurrent sepsis, kidney obstruction, cyst rupture and, very rarely, small renal cancer picked up on CT scan. Massive hepatomegaly may lead to increased intraabdominal pressure, compression of the diaphragm and stomach resulting in reduced exercise tolerance, breathlessness, early satiety and malnutrition, portal hypertension and ascites. Brain aneurysms may rupture leading to intracranial bleeding and stroke.

Genetic and clinical predictors of rapid deterioration of kidney function include truncating PKD1 mutation, decreased glomerular filtration rate (GFR) at age $<35$ years, increased kidney size, hypertension and urological complications at age $<35$ years, male gender and family history of ESRF at age $<55$ years [1]. Patients with ADPKD with a family history of SAH or stroke are likely to be screened for berry aneurysm as the prevalence of intracranial aneurysm may be as high as $25 \%$. Brain CT angiogram to monitor berry aneurysm and the need for early clipping to prevent bleeding are paramount.

\section{Treatment}

There is no cure available for this condition. A new drug, Tolvaptan (Vasopressin V2 receptor antagonist) is showing promise by slowing down the formation and growth 
of cysts and progression to renal failure. It is recommended for use at an early stage of renal dysfunction, GFR $>60$. It can delay the $4-6 \mathrm{~mL} / \mathrm{min} / 1.73 \mathrm{~m}^{2}$ per year fall in GFR often observed in affected ADPKD patients [2]. However, idiosyncratic hepatocellular toxic effects and the often reported polyuria, polydipsia, nocturia, and urinary frequency are a drawback for its use. The longterm effectiveness of treatment using Tolvaptan remains to be determined. Generally, the objective is to delay the onset of ESRF, treat symptoms and complications and perform pre-emptive kidney transplantation or dialysis. Simple, percutaneous drainage of large, symptomatic accessible cysts may be undertaken. Rarely, massively enlarged failed liver may warrant liver transplantation.

\section{Surgical management}

Pre-emptive kidney transplant, transplant done before dialysis is necessary, is the best treatment option with associated better patient and allograft survival compared with patients with other causes of ESRF [3]. Early diagnosis and observed gradual progression of renal failure make pre-emptive transplantation more likely.

\section{Native nephrectomy}

Removal of one or both native kidneys before or after transplant is not required in most patients, because affected patients want to stay off dialysis for as long as possible and be able to continue urinating while on dialysis, making fluid restriction and possibly renal anaemia easier to manage. If the indication for nephrectomy is to create space for a transplant kidney, only one kidney needs to be removed.

Bilateral nephrectomies are associated with higher risks of morbidity and mortality. Such patients lose out in three ways if both kidneys are removed. There is a higher chance that blood transfusion will be required, which may result in sensitisation of the potential transplant recipient, making finding a kidney more challenging. Furthermore, both adrenal glands may be damaged during bilateral nephrectomies resulting in hypotension requiring shortor long-term drug treatment. Bilateral nephrectomies lead to loss of erythropoietin (EPO)-producing cells making anaemia inevitable. Therefore, bilateral nephrectomies are only indicated if both kidneys pose a threat to the patient's life, adversely affect the quality of life, or the transplanted kidney, or if they harbour foci of infection or cancer.

\section{Summary}

ADPKD is an inherited cause of kidney failure affecting all races. The onset of symptoms and severity of the disease vary in affected individuals. Less commonly, organs such as the brain, liver, pancreas, spleen, heart and blood vessels may have associated pathologies leading to complications. The gradual unrelenting enlargement of the kidney and liver poses significant lifestyle challenges for affected patients. The widespread use of the new agent, Tolvaptan, is limited by its hepatoxic side effects. We are yet to learn how best to select patients who will benefit from long-term treatment using this drug. Pre-emptive kidney transplantation is the best treatment option for this group of patients. The large, symptomatic polycystic kidney may need to be removed surgically to create space for a kidney transplant or removed after successful kidney transplant if they cause severe, distressing symptoms or threaten the life of patient or allograft survival or quality of life of the patient.

\section{Conflict of interest and funding}

The author has not received any funding or benefits from industry or elsewhere to conduct this study.

\section{References}

1. Cornec-Le Gall E, Audrézet M-P, Rousseau A, Hourmant M, Renaudineau E, Charasse C, et al. The PROPKD score: A new algorithm to predict renal survival in autosomal dominant polycystic kidney disease. J Am Soc Nephrol 2016; 27: 942-51. doi: 10.1681/ASN.2015010016

2. Torres VE, Chapman, AB, Devuyst $\mathrm{O}$, et al. Tolvaptan in later-stage autosomal dominant polycystic kidney disease. N Engl J Med 2017; 377: 1930-42. doi: 10.1056/NEJMoa1710030

3. Jacquet A, Pallet N, Kessler M. Outcomes of renal transplantation in patients with autosomal dominant polycystic kidney disease: a nationwide longitudinal study. Transpl Int 2011; 24: 582-7. doi: 10.1111/j.1432-2277.2011.01237.x 